\title{
Probing Electron-Phonon Interactions Away from the Fermi Level with Resonant Inelastic X-Ray Scattering
}

C. D. Dashwood $\odot,{ }^{1, *}$ A. Geondzhian, ${ }^{2,3, \ddagger}$ J. G. Vale, ${ }^{1}$ A. C. Pakpour-Tabrizi ${ }^{4}$ C. A. Howard $\odot,{ }^{1}$ Q. Faure, ${ }^{1}$ L. S. I. Veiga, ${ }^{1}$ D. Meyers, ${ }^{5,6}$ S. G. Chiuzbăian $\odot,{ }^{7,8}$ A. Nicolaou $\odot,{ }^{7}$ N. Jaouen $\odot,{ }^{7}$ R. B. Jackman $\odot,{ }^{4}$ A. Nag $\odot,{ }^{9}$ M. García-Fernández, ${ }^{9}$ Ke-Jin Zhou, ${ }^{9}$ A. C. Walters $\odot,{ }^{9}$ K. Gilmore $\odot,{ }^{5,10,11}$ D. F. McMorrow, ${ }^{1}$ and M. P. M. Dean $\odot^{5, \uparrow}$

${ }^{1}$ London Centre for Nanotechnology and Department of Physics and Astronomy, University College London, London, WC1E 6BT, United Kingdom

${ }^{2}$ Max Planck POSTECH/KOREA Research Initiative, 37673 Pohang, South Korea

${ }^{3}$ Max Planck Institute for the Structure and Dynamics of Matter, Luruper Chaussee 149, 22761 Hamburg, Germany

${ }^{4}$ London Centre for Nanotechnology and Department of Electronic and Electrical Engineering, University College London, London, WC1E 6BT, United Kingdom

${ }^{5}$ Condensed Matter Physics and Materials Science Department, Brookhaven National Laboratory, Upton, New York 11973, USA

${ }^{6}$ Department of Physics, Oklahoma State University, Stillwater, Oklahoma 74078, USA

${ }^{7}$ Synchrotron SOLEIL, L'Orme des Merisiers, Saint-Aubin, B.P. 48, 91192 Gif-sur-Yvette, France

${ }^{8}$ Sorbonne Université, CNRS, Laboratoire de Chimie Physique-Matiére et Rayonnement, UMR 7614, 4 place Jussieu, 75252 Paris Cedex 05, France

${ }^{9}$ Diamond Light Source, Didcot, Oxfordshire, OX11 ODE, United Kingdom

${ }^{10}$ Physics Department and IRIS Adlershof, Humboldt-Universität zu Berlin, Zum Großen Windkanal 2, 12489 Berlin, Germany

${ }^{11}$ European Theoretical Spectroscopy Facility (ETSF)

(Received 10 May 2021; revised 18 October 2021; accepted 20 October 2021; published 15 December 2021)

Interactions between electrons and lattice vibrations are responsible for a wide range of material properties and applications. Recently, there has been considerable interest in the development of resonant inelastic x-ray scattering (RIXS) as a tool for measuring electron-phonon ( $e$-ph) interactions. Here, we demonstrate the ability of RIXS to probe the interaction between phonons and specific electronic states both near to, and away from, the Fermi level. We perform carbon $K$-edge RIXS measurements on graphite, tuning the incident x-ray energy to separately probe the interactions of the $\pi^{*}$ and $\sigma^{*}$ electronic states. Our high-resolution data reveal detailed structure in the multiphonon RIXS features that directly encodes the momentum dependence of the $e$-ph interaction strength. We develop a Green's-function method to model this structure, which naturally accounts for the phonon and interaction-strength dispersions, as well as the mixing of phonon momenta in the intermediate state. This model shows that the differences between the spectra can be fully explained by contrasting trends of the $e$-ph interaction through the Brillouin zone, being concentrated at the $\Gamma$ and $K$ points for the $\pi^{*}$ states while being significant at all momenta for the $\sigma^{*}$ states. Our results advance the interpretation of phonon excitations in RIXS and extend its applicability as a probe of $e$-ph interactions to a new range of out-of-equilibrium situations.

DOI: $10.1103 /$ PhysRevX.11.041052

Subject Areas: Condensed Matter Physics

*cameron.dashwood.17@ucl.ac.uk

†mdean@bnl.gov

These authors contributed equally to this work.

Published by the American Physical Society under the terms of the Creative Commons Attribution 4.0 International license. Further distribution of this work must maintain attribution to the author(s) and the published article's title, journal citation, and DOI.

\section{INTRODUCTION}

Knowledge of the interactions between electrons and phonons is central to understanding a diverse array of condensed matter phenomena. Basic material properties such as low-field electrical transport [1], as well as emergent collective phases including charge density waves [2] and conventional superconductivity [3], are all crucially dependent on the coupling of phonons to electrons near the Fermi surface. Extensive experimental efforts have, therefore, been devoted to quantifying this coupling through various techniques. Neutron scattering [4], nonresonant 
inelastic x-ray scattering (IXS) [5], and Raman spectroscopy [6] all probe $e$-ph coupling via the lattice degrees of freedom, while angle-resolved photoemission spectroscopy (ARPES) [7] measures it via the electronic self-energy.

In other far-from-equilibrium situations, however, the interaction between phonons and electrons away from the Fermi surface becomes important. This regime applies to processes such as high-temperature heat transport [8], highfield electrical transport [9], and phonon-assisted optical transitions $[10,11]$, with applications in optoelectronics $[12,13]$, for example. The established probes of $e$-ph coupling, listed above, are generally unable to access such highly excited electronic states, particularly if information is required about the interaction with phonon modes of specific momenta.

Recently, resonant inelastic x-ray scattering (RIXS) has been gaining traction as a new technique to measure the $e$-ph interaction strength, which manifests directly in the intensity of the phonon excitations. Over the past decade, improvements in energy resolution have enabled experimental studies on a growing number of materials [14-27], alongside an advancing theoretical understanding of the phonon contribution to the RIXS cross section [28-32]. Much of the excitement around these developments lies in the momentum resolution that RIXS offers. The other great strength of RIXS - the ability to probe a particular electronic orbital by tuning the incident energy to a specific absorption edge - has thus far been underexploited.

Here, we demonstrate the power of RIXS to probe the interaction of phonons with two distinct electronic states in graphite: the $\pi^{*}$ state near the Fermi level and the $\sigma^{*}$ state well above it. At the resonances of these two states, we find qualitatively different multiphonon excitations in our RIXS spectra, showing a stark difference in the phonon momenta to which they couple. This behavior cannot be captured by the currently available theoretical models, which assume dispersionless phonons $[19,28,32]$ or focus on one-phonon processes [29]. We, therefore, extend a Green's-function approach, previously applied to small molecules [30], to treat the full momentum dependence of phonons in a crystalline lattice. This model accurately reproduces our experimental spectra at both resonances, revealing contrasting trends of the $e$-ph interaction strength through the Brillouin zone.

\section{RIXS MEASUREMENTS ON GRAPHITE}

Graphite is a favorable material for this study, as it has a simple electronic and phononic structure as well as two well-defined excitations to the low-energy $\pi^{*}$ and highenergy $\sigma^{*}$ states at the carbon $K$ edge. Coupling to the electronic states near the Fermi level is known to be dominated by the highest optical phonon band above $0.15 \mathrm{eV}$ [5,6,33-35], which is well resolved by our 47-meV-resolution measurements. There are also no other low-energy excitations (such as magnons) that could obscure the phonon excitations in our RIXS spectra.
This allows us to validate our methodology at the $\pi^{*}$ resonance, before extending our treatment to the $\sigma^{*}$ states. Given their similarities to graphite $[6,33,34]$, our results are also applicable to the other technologically important allotropes of carbon.

Our carbon $K$-edge RIXS measurements on graphite are summarized in Fig. 1(a), which shows approximately zonecenter RIXS maps around the $\pi^{*}$ and $\sigma^{*}$ resonances (see the Appendix A for experimental details). Above the elastic line at $0 \mathrm{eV}$ energy loss, both maps show a series of features of decreasing intensity that are reminiscent of the harmonic progression of phonon excitations seen in previous studies [15-27]. These features are more intense in the $\sigma^{*}$ map, suggesting a stronger overall coupling.

We assign contributions to the phonon features through reference to the known phonon dispersion of graphite, shown in Fig. 1(b) [36]. The lowest-energy feature occurs at approximately $0.19 \mathrm{eV}$ in both maps, which corresponds to the degenerate transverse (TO) and longitudinal optical (LO) modes at the $\Gamma$ point [whose displacement pattern is

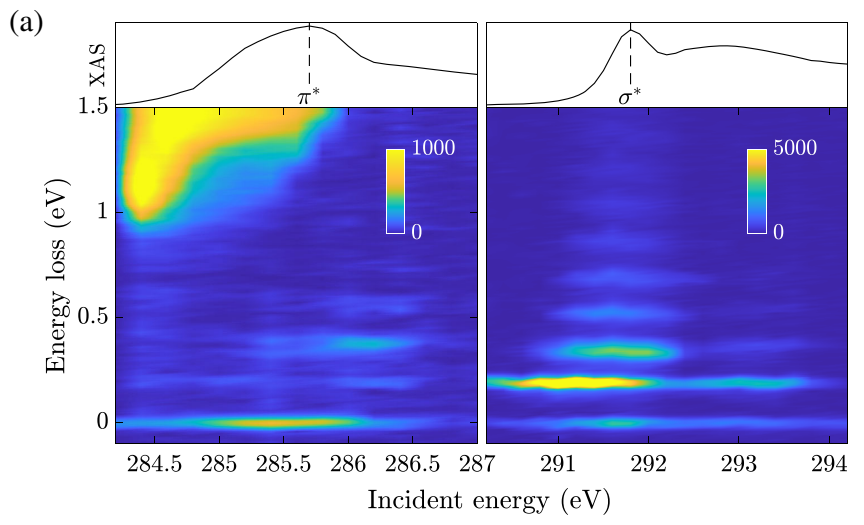

(b)

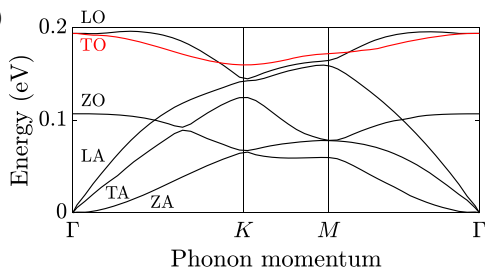

(c)

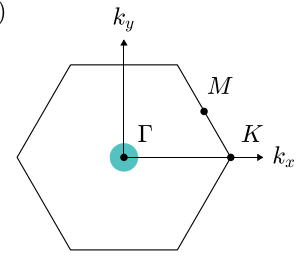

FIG. 1. Contrasting RIXS response at the $\pi^{*}$ and $\sigma^{*}$ resonances. (a) RIXS maps around both resonances, with $\mathrm{x}$-ray absorption spectroscopy (XAS) above for reference (the dashed vertical lines in the XAS mark the peaks of the resonances at 285.6 and $298.1 \mathrm{eV}$, respectively). Both maps show a series of phonon features above the elastic line, with the contrasting resonance behavior most apparent for the two-phonon feature between 0.3 and $0.4 \mathrm{eV}$. The intense feature above approximately $1 \mathrm{eV}$ in the $\pi^{*}$ map arises from electronic transitions. (b) Phonon dispersion of graphite from Ref. [36], with the transverse $(T)$, longitudinal $(L)$, and out-of-plane $(Z)$ acoustic $(A)$ and optical $(O)$ modes marked. The TO mode with significant coupling is marked in red. (c) The 2D-projected Brillouin zone of graphite, with the momenta accessible in our RIXS measurement indicated by the turquoise circle and high-symmetry positions $\Gamma, K$, and $M$ marked. 

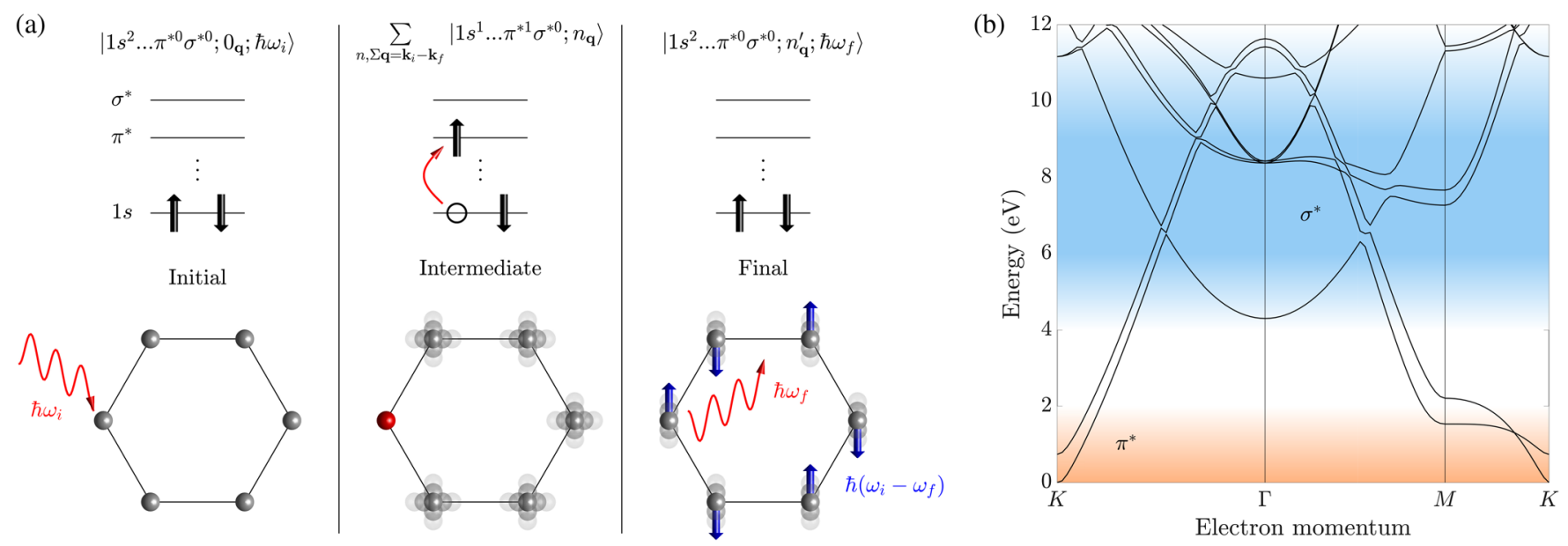

FIG. 2. Phonon generation in RIXS. (a) Schematic of the RIXS process at the carbon $K$ edge. An incident photon of energy $\hbar \omega_{i}$ and wave vector $\boldsymbol{k}_{i}$ excites an electron from the $1 s$ to the $\pi^{*}$ or $\sigma^{*}$ bands, leaving a core hole. In the intermediate state, the altered charge density perturbs the positions of surrounding ions and generates $n_{q}$ phonon modes. The excited electron then relaxes via the emission of a photon of energy $\hbar \omega_{f}$, leaving $n_{\boldsymbol{q}}^{\prime}$ phonons with total energy $\hbar\left(\omega_{i}-\omega_{f}\right)$ in the final state (the zone-center TO mode is depicted [36]). (b) Electronic band structure of graphite from Ref. [37], with the low-energy $\pi^{*}$ bands shaded in orange and the high-energy $\sigma^{*}$ bands in blue.

shown in the final state in Fig. 2(a)]. In a purely local picture, successive peaks would then correspond to higher harmonics of this first peak. The next feature around 0.3-0.4 eV energy loss, however, shows an internal structure that differs markedly between the two maps. At the $\pi^{*}$ resonance $(285.6 \mathrm{eV}$ ) the feature is split into two (as can also be seen in the line cuts in Fig. 3), while at the $\sigma^{*}$ resonance $(291.8 \mathrm{eV})$ a single broader asymmetric peak is visible.

To conserve momentum, a single-phonon excitation in a zone-center spectrum can include only a zone-center mode in the final state. The final states of a two-phonon feature, however, can conceivably consist of pairs of oppositemomenta phonons at any point in the Brillouin zone. The split feature at the $\pi^{*}$ resonance has peak energies of 0.32 and $0.39 \mathrm{eV}$, which correspond to double the energies of the TO mode at $K$ and $\Gamma$, respectively (see Supplemental Material [38]). Meanwhile, the broad feature at the $\sigma^{*}$ resonance spans double the full bandwidth of the TO mode. Phonons of different momenta, therefore, appear to be excited at each of the resonances.

\section{ELECTRON-PHONON INTERACTIONS IN RIXS}

We have identified the modes that contribute to the RIXS spectra, but why do phonons of different momenta appear at the $\pi^{*}$ and $\sigma^{*}$ resonances? To answer this, we need to understand how phonons are generated in a RIXS measurement.

Figure 2(a) shows a schematic of the RIXS process at the carbon $K$ edge. An incident x-ray photon excites an electron from the $1 s$ to the $\pi^{*}$ or $\sigma^{*}$ bands, depending on the energy, where it experiences a partially screened potential from the resulting core hole. The altered charge density of this intermediate state perturbs the positions of the surrounding ions, generating multiple phonons. Finally, the excited electron relaxes to fill the core hole through the emission of a photon, with the difference in incident and scattered photon energies equal to the energy of the phonons remaining in the final state.

The composition of the intermediate electronic state can, therefore, have a significant impact on the phonon modes that are excited. As both resonances involve the same core hole, this cannot explain the difference between the RIXS spectra. We instead focus on the band structure of the excited electron, shown in Fig. 2(b). For electrons excited into the low-energy $\pi^{*}$ states, the steepness of the bands confines them to a small region around the Dirac points at $K$. These states can interact only with phonons with momenta close to $\Gamma$, which scatter electrons within a single Dirac cone, or $K$, which scatter electrons between different Dirac cones. By contrast, the high-energy $\sigma^{*}$ bands are flatter and electrons excited into them can span the Brillouin zone, suggesting interactions with a wide range of phonon momenta. This provides an intuitive understanding of our earlier assignment of the RIXS features, where the split two-phonon feature at the $\pi^{*}$ resonance is dominated by phonons at $\Gamma$ and $K$, while the single broad feature at the $\sigma^{*}$ resonance has contributions from across the TO mode dispersion.

To put this on a more rigorous footing, we need a theoretical model that can account for interactions of the full excitonic intermediate state with multiple phonons of different momenta. Within the exact-diagonalization approaches proposed thus far [19,28,29], this requires a computationally intractable expansion of the Hilbert space. We, therefore, employ a Green's-function approach which 
implicitly accounts for vibronic effects in the intermediate state (full details can be found in Appendix B). We address $e$-ph interactions within the framework of a linked-cluster (cumulant) expansion using a second-order Fan-Migdaltype self-energy in the time domain. RIXS cross sections are obtained by considering the nondiagonal elements of an exciton Green's function, as described elsewhere [30]. As well as fully accounting for the phonon dispersion, this approach allows a proper treatment of mode mixing in the intermediate state, which leads to significant renormalization of the spectral features.

In order to focus on the effect of the interaction-strength dispersion, we treat both $\pi^{*}$ and $\sigma^{*}$ intermediate states as localized excitons, which is a good approximation over the intermediate-state lifetime of graphite [39]. The intermediate-state lifetime itself is determined using the core-hole lifetime from x-ray photoemission spectroscopy [40], adjusted to mimic the presence of the extra electron (see Supplemental Material [38]). Given the weak interlayer coupling of graphite, the optical phonon modes of interest have a minimal dependence on the out-of-plane momentum [36], and we can safely restrict our analysis to the 2Dprojected Brillouin zone. We use the phonon dispersion calculated with density functional perturbation theory, which is in good agreement with IXS measurements [36]. The only free parameter of the model is then the momentum-dependent $e$-ph interaction strength $G(\boldsymbol{q})$, which can be determined by fitting the experimental RIXS spectra.

\section{ELECTRON-PHONON INTERACTIONS NEAR THE FERMI LEVEL}

We turn first to our data around the $\pi^{*}$ resonance. From our earlier assignment of the RIXS features, it appears that significant contributions come only from the TO mode at the $K$ point and degenerate TO and LO modes at the $\Gamma$ point (see Supplemental Material [38]). We, therefore, include only the TO mode dispersion in our modeling, with a minimal set of momentum points: the $\Gamma$ and $K$ points, the experimental inplane momentum transfer $\boldsymbol{q}_{\exp } \approx(0.08,0.00)$ r.l.u., and $K-\boldsymbol{q}_{\text {exp }}$. Inclusion of the latter two effectively captures the width over which $G(\boldsymbol{q})$ falls off away from the highsymmetry points. The intensity of the one-phonon peak is sensitive to the fact that $\boldsymbol{q}_{\exp } \neq \Gamma$, but for the multiphonon features it is sufficient to approximate the total momentum transfer as zero.

Figure 3(b) shows a fit of this model to the spectrum on resonance, with the corresponding momentum dependence of $G(\boldsymbol{q})$ plotted in Fig. 3(a). We see good agreement between the experimental and calculated spectra, including the striking split two-phonon feature, despite our simple parameterization of $G(\boldsymbol{q})$. This confirms that the splitting arises due to the dominant contributions from pairs of phonons at $K$ and $\Gamma$, indicated by the vertical lines at 0.32 and $0.39 \mathrm{eV}$, respectively. Intensity above $0.45 \mathrm{eV}$ is attributed to overlapping three- and four-phonon features, which are also consistent with contributions coming from pairs of $\pm K$-momenta phonons with additional $\Gamma$ modes.

We further test our model through its ability to reproduce spectra with the incident $\mathrm{x}$-ray energy detuned from the maximum of the XAS. Figure 3(c) shows the energy dependence of the experimental and calculated spectra through the resonance. As well as the persistent splitting of the two-phonon feature below the resonance, our model captures the changing relative intensities of the one- and two-phonon features. The nonmonotonic energy dependence of these intensities, including the offset of the
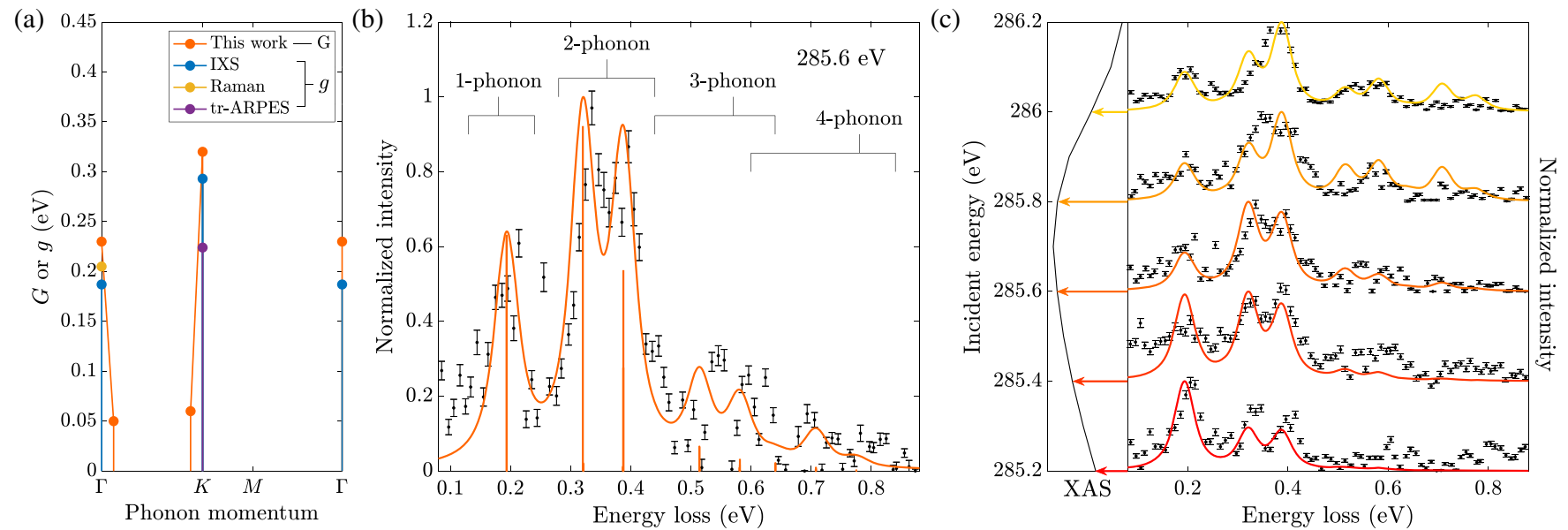

FIG. 3. $e$-ph interactions for low-energy $\pi^{*}$ excitations. (a) Modeled momentum dependence of $G$ through the 2D Brillouin zone (orange), compared to $g$ determined by IXS (blue) [5], Raman spectroscopy (yellow) [6], and time-resolved ARPES (purple) [35]. (b) Normalized experimental (black points with error bars) and calculated (orange line, including experimental broadening) RIXS spectra on resonance $(285.6 \mathrm{eV})$. The contribution from each phonon generation process is indicated by the vertical orange lines, grouped above by the number of phonons. (c) Incident-energy dependence of the experimental (black points with error bars) and calculated (red to orange lines) spectra, plotted alongside the XAS with arrows indicating the energies. 
maximum of the one-phonon peak intensity from zero detuning [27], arises from a combination of strong $e$-ph interaction strength and long core-hole lifetime [31]. We emphasize that the only parameter being varied between the spectra in Fig. 3(c) is the incident energy, providing a strong validation of our model.

As the incident energy is raised through the upper tail of the XAS peak, the composition of the intermediate electronic state changes [picking up contributions from the $M$ point; see Fig. 2(b)] [41]. This, in turn, allows scattering by phonons of different momenta, changing the momentum dependence of $G$ and explaining the discrepancies between the experimental and calculated spectra above $285.6 \mathrm{eV}$. Agreement can be improved by allowing $G(\boldsymbol{q})$ to vary slightly with incident energy (see Supplemental Material [38]).

We can now compare our results with those from other techniques. Alongside our best-fit $G(\boldsymbol{q})$, Fig. 3(a) also shows the $e$-ph interaction strength determined by IXS [5] and Raman spectroscopy [6], as well as a novel timeresolved ARPES technique [35]. We see reasonable agreement between all the techniques. Note, however, that we use a lowercase $g$ for the coupling probed by these other techniques, to highlight that they do not involve a core hole. As discussed above, RIXS measures a related but inequivalent coupling $G$ of phonons to an excitonic intermediate state consisting of both the excited electron and core hole [30]. The concept of exciton-phonon coupling is not new, being relevant to Raman and other optical spectroscopies $[42,43]$ (the only difference here being that the hole is often in the valence band rather than core level). Recent work in these fields shows that, under certain approximations, the two quantities $G$ and $g$ can be related by [44]

$$
G_{n m}^{\nu}(\boldsymbol{Q}, \boldsymbol{q})=\sum_{\boldsymbol{k}}\left[\sum_{h, c, c^{\prime}} A_{h(\boldsymbol{k}), c(\boldsymbol{k}+\boldsymbol{Q}+\boldsymbol{q})}^{m(\boldsymbol{Q}+\boldsymbol{q}) *} A_{h(\boldsymbol{k}), c^{\prime}(\boldsymbol{k}+\boldsymbol{Q})}^{n(\boldsymbol{Q})} g_{c^{\prime} c}^{\nu}(\boldsymbol{k}+\boldsymbol{Q}, \boldsymbol{q})-\sum_{c, h, h^{\prime}} A_{h(\boldsymbol{k}-\boldsymbol{q}), c(\boldsymbol{k}+\boldsymbol{Q})}^{m(\boldsymbol{Q}+\boldsymbol{q}) *} A_{h^{\prime}(\boldsymbol{k}), c(\boldsymbol{k}+\boldsymbol{Q})}^{n(\boldsymbol{Q})} g_{h h^{\prime}}^{\nu}(\boldsymbol{k}-\boldsymbol{Q}, \boldsymbol{q})\right] .
$$

This expression describes an exciton in initial state $n$, with center-of-mass momentum $\boldsymbol{Q}$, and wave function $\left|S_{n}\right\rangle=\sum_{h c} A_{h c}^{n}|h c\rangle$, where $h(c)$ indicate hole (conduction) states. The exciton is scattered by a phonon in mode $\nu$ with momentum $\boldsymbol{q}$, to a final state $m$ with momentum $\boldsymbol{Q}+\boldsymbol{q}$ and wave function $\left|S_{m}\right\rangle=\sum_{h^{\prime} c^{\prime}} A_{h^{\prime} c^{\prime}}^{m}\left|h^{\prime} c^{\prime}\right\rangle$. It can be seen that Eq. (1) is separated into two terms, the first accounting for electron-phonon scattering with the hole state unaffected and the second for hole-phonon scattering with a passive electron, each weighted by the coefficients of the excitonic wave function. In cases where the core hole is strongly screened, RIXS reflects the single-particle coupling of the excited electron. When the core hole is weakly screened, RIXS accesses exciton-phonon coupling, which is the quantity of interest for the many out-of-equilibrium situations discussed in the introduction.

From Fig. 3(a), we can also see how the various techniques probe different components of $g_{c c^{\prime}}^{\nu}(\boldsymbol{Q}, \boldsymbol{q})$ and $G_{n m}^{\nu}(\boldsymbol{Q}, \boldsymbol{q})$. Standard ARPES resolves $g$ by electronic momentum $\boldsymbol{Q}$ for bands $c$ below the Fermi energy but integrates over phonon modes $\nu$ and momenta $\boldsymbol{q}$ [7]. By contrast, IXS [5] and neutron scattering [4] resolve the phonon momentum $\boldsymbol{q}$ throughout the Brillouin zone for all modes $\nu$ but average over the equilibrium electronic state. Raman spectroscopy [6] and the novel time-resolved ARPES [35] technique can access low-energy electronic excitations but are restricted to particular phonon momenta $(\boldsymbol{q} \approx \Gamma$ and $K$ for graphite). By contrast, we show that RIXS can resolve $G$ by electronic state $n$, phonon mode $\nu$, and phonon momentum $\boldsymbol{q}$, integrating only over the electronic momentum $\boldsymbol{Q}$.
By modeling the contributions to the multiphonon features, we therefore determine the interactions between the $\pi^{*}$ electronic state and TO phonons throughout the Brillouin zone, all from a single zone-center spectrum. This method will be invaluable, as the soft $\mathrm{x}$-ray edges at which phonons are enhanced (due to the long intermediate-state lifetimes) and energy resolutions are maximized also suffer from restricted momentum transfer [see Fig. 1(c)].

\section{ELECTRON-PHONON INTERACTIONS AWAY FROM THE FERMI LEVEL}

Having successfully reproduced the $\pi^{*}$ spectra, we now come to the high-energy $\sigma^{*}$ states. Based on our assignment of the RIXS features, we again restrict our analysis to the TO mode (see Supplemental Material [38]). In this case, however, the broad profile of the two-phonon peak suggests $G(\boldsymbol{q})$ is finite over a wide range of momenta. In order to constrain our parameter space, we therefore assume a linear dependence between the high-symmetry points $\Gamma, K$, and $M$. Given the more uniform variation of $G(\boldsymbol{q})$ around $\Gamma$, we can also approximate the experimental momentum transfer as zero for the whole spectrum.

Figure 4(a) shows the best-fit dispersion of $G(\boldsymbol{q})$, which we find to be significant at all the high-symmetry points. The resulting spectrum on resonance is shown in Fig. 4(b), and again we see that a simple parameterization of reciprocal space is able to describe the experimental data well. Unlike the splitting seen at the $\pi^{*}$ resonance, here our model reproduces the subtle broadening and asymmetry of the two-phonon feature, confirming that these characteristics arise from the varying contributions of pairs of 

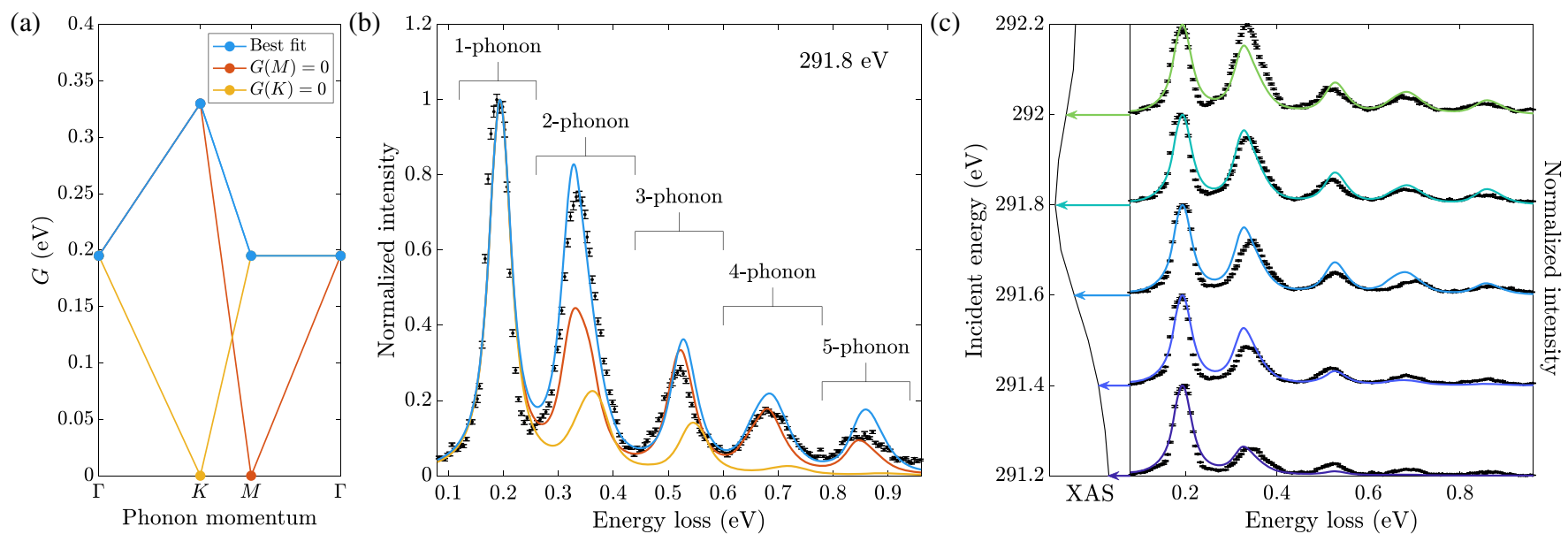

FIG. 4. $e$-ph interactions for high-energy $\sigma^{*}$ excitations. (a) Best-fit momentum dependence of $G$ (blue line) and that with $G(M)=0$ (orange line) and $G(K)=0$ (yellow line). (b) Normalized experimental (black points with error bars) and calculated [blue, orange, and yellow lines, corresponding to those in (a)] RIXS spectra on resonance (291.8 eV). The contribution from each $n$-phonon process is labeled above. (c) Incident-energy dependence of the experimental (black points with error bars) and calculated (blue to green lines) spectra, plotted alongside the XAS with arrows indicating the energies.

opposite-momenta phonons which disperse gradually through the Brillouin zone.

In order to determine the sensitivity of our fit to the values at the high-symmetry points, we also show spectra in Fig. 4(b) calculated with $G(M)=0$ and $G(K)=0[G(\Gamma)$ must be finite to produce a significant one-phonon peak]. We can see that the calculated spectra are most sensitive to the value of $G(K)$, which strongly influences the intensities of all the multiphonon peaks. While the value of $G(M)$ has little impact on the three-, four-, and five-phonon peaks, we see that a finite value on the order of $G(\Gamma)$ is required to accurately capture the intensity and shape of the twophonon peak. We also show in Supplemental Material [38] how a previous RIXS measurement of $G(\boldsymbol{q})$ at the $\sigma^{*}$ resonance of graphite [27], which assumes zero coupling between the high-symmetry points, is unable to reproduce the multiphonon features.

Our best-fit $G(\boldsymbol{q})$ is also able to reproduce the changing intensities of the features on detuning below $291.8 \mathrm{eV}$, as shown in Fig. 4(c). As for the $\pi^{*}$ states, we expect agreement between the model and experiment to degrade above the resonance due to changes in the intermediate electronic state. Furthermore, just above the $\sigma_{1}^{*}$ resonance, that we are focused on, lies the $\sigma_{2}^{*}$ resonance [see Fig. 2(b)], which arises from zero-point vibrations [41] that are not included in our modeling.

\section{CONCLUSION}

We have demonstrated that, through careful choice of the incident photon energy, RIXS is able to probe the interactions between phonons and electrons both close to, and away from, the Fermi level. Our results for graphite reveal that the momentum dependence of the interaction strength, $G(\boldsymbol{q})$, can be highly distinct in these two regimes: For low-energy $\pi^{*}$ states it is concentrated in small regions around the $\Gamma$ and $K$ points, while for high-energy $\sigma^{*}$ states it is significant across the Brillouin zone. This establishes RIXS as a uniquely versatile probe of $e$-ph interactions in crystalline materials, applicable to studies of out-ofequilibrium processes alongside ordered phases in correlated systems. Combined with the recent development of ultrafast time-resolved RIXS at $\mathrm{x}$-ray free-electron lasers [45-48], our methodology should prove particularly powerful for determining $e$-ph interactions in pumped systems.

We find that contributions to the multiphonon features in a RIXS spectrum can come from phonons away from the experimental momentum transfer, allowing us to constrain $G(\boldsymbol{q})$ throughout the Brillouin zone from a single zonecenter spectrum. This is particularly apparent at the $\pi^{*}$ resonance, where the rapid variation of $G$ with momentum produces a split two-phonon feature. These contributions are also important, however, for the $\sigma^{*}$ states with more uniform $G(\boldsymbol{q})$. Here, the gradually dispersing phonons cause a broadening and asymmetry of the multiphonon features, as has been seen in other studies [15,22], alongside shifts in their peak energies that could be mistaken for anharmonic effects [22]. As we show in Appendix B, mode mixing in the intermediate state results in the multiphonon contributions affecting the intensity and resonance behavior of all the phonon features in the spectra, including the one-phonon peak. Neglecting this effect is likely to lead to qualitative errors in the determination of $G(\boldsymbol{q})$ [27]. These insights from graphite will be important when analyzing the phonon excitations in RIXS measurements of more complex materials, such as the much-studied cuprates [14-16,19,21,23,25,26].

Our results highlight the importance of developing a robust theoretical description of the complex RIXS process in order to analyze experimental data. The Green's-function 
approach that we employ offers a practical way to incorporate the phonon and $e$-ph interaction-strength dispersions without the need for summation over all possible intermediate states. There are many avenues that remain to be explored, including the effects of itinerancy [32] and changes to the potential energy surface [30] in the intermediate state and how the core hole affects the coupling probed by RIXS compared to other techniques [30]. The availability of theoretical methods to treat these effects will only become more pressing as the energy resolution of RIXS improves, and it is used to probe phonons in an everincreasing range of topical materials.

\section{ACKNOWLEDGMENTS}

We thank Matteo Calandra for insightful discussions. C.D. D. was supported by the Engineering and Physical Sciences Research Council (EPSRC) Centre for Doctoral Training in the Advanced Characterisation of Materials under Grant No. EP/L015277/1. Work at Brookhaven National Laboratory was supported by the U.S. Department of Energy, Office of Science, Office of Basic Energy Sciences, under Contract No. DE-SC0012704. A. G. was supported by a National Research Foundation of Korea grant funded by the Ministry of Science and ICT No. 2016K1A4A4A01922028 and the U.S. National Science Foundation under Grant No. DMR-1904716 and acknowledges Research Computing at Arizona State University for providing high-performance computing resources. K. G. was supported by the U.S. Department of Energy, Office of Science, Basic Energy Sciences as part of the Computational Materials Science Program through the Center for Computational Design of Functional Strongly Correlated Materials and Theoretical Spectroscopy. R. B. J. acknowledges the support of the European Commission "GREENDIAMOND" project through the H2020 Large Project under Grant No. SEP-210184415. Work at U.C.L. was supported by the EPSRC under Grants No. EP/N027671/1, No. EP/H020055/1, and No. EP/ N004159/1. We acknowledge SOLEIL for provision of synchrotron radiation facilities at the SEXTANTS beam line under Proposals No. 20150221 and No. 20130732 and Diamond Light Source for time on beam line I21 under proposal MM22695.

\section{APPENDIX A: EXPERIMENTAL DETAILS}

Our carbon $K$-edge RIXS measurements were performed at beam line I21 at Diamond Light Source. A natural graphite single crystal was mounted such that the scattering plane is $(h, 0, l)$ and cleaved in vacuum. We chose an energy resolution of $47 \mathrm{meV}$ (determined from scattering from amorphous carbon tape mounted next to the sample) to balance throughput and resolution. All RIXS spectra were taken with linear horizontal $(\pi)$ incident $\mathrm{x}$-ray polarization, at a temperature of $20 \mathrm{~K}$, and with a counting time of 20 min. X-ray absorption spectra in total electron yield were obtained by measuring the sample drain current. Data for energies around the $\pi^{*}$ resonance were taken with grazing-incident $\mathrm{x}$ rays $\left(\theta=20^{\circ}\right)$, while data around the $\sigma^{*}$ resonance were taken with normal-incident $\mathrm{x}$ rays $\left(\theta=90^{\circ}\right)$ in order to maximize the intensity of the phonon features. All data are corrected for incident flux and selfabsorption (see Supplemental Material [38]).

At these soft $\mathrm{x}$-ray energies, the momentum transfer is confined to a small in-plane region of $<0.1$ r.l.u. around the zone center [see Fig. 1(c)]. As we use a fixed scattering angle $2 \Theta=154^{\circ}$, the momentum transfer varies slightly with incident energy. Around the $\pi^{*}$ resonance the momentum transfer $\boldsymbol{q}=(0.0802 \pm 0.0004,0,0.164 \pm 0.001)$, while around the $\sigma^{*}$ resonance $\boldsymbol{q}=(0.0220 \pm 0.0002,0$, $0.300 \pm 0.002)$.

\section{APPENDIX B: THEORETICAL MODEL}

To model our rich experimental data, we employ a phenomenological Green's-function formulation of the RIXS cross section. In principle, final states involving both phonons and low-energy electronic excitations are allowed, the latter of which result in a continuum of excitations. As such a continuum is not seen above the background in our data, however, we neglect these weak higher-order process and restrict the final states to those with only phonon excitations present.

We account for electron-electron interactions by constructing an effective quasiparticle exciton Green's function. Because of the different timescales, it is reasonable to separately consider electron-lattice interactions in the presence of an exciton which propagates through the material $[30,43]$. Expanding the interacting exciton Green's function in a time series, we find two important types of diagrams: closed-loop diagrams, where a phonon gets created and destroyed by the same exciton, and those where a phonon connects two excitons and gives rise to the final state phonon population. The former affect the intensities of the RIXS features nonlinearly and can be interpreted as contributions from intermediate-state phonons. Evaluating these contributions has proven to be very computationally demanding in exact-diagonalization approaches. Here, we account for them using the cumulant representation of the interacting Green's function, which reduces computational cost. The second type of diagram is treated explicitly up to the number of phonon satellites visible in the experiment.

To simplify the notation, we omit electron-photon matrix elements, which affect only the absolute intensity and not the characteristic shape of the phonon features, and drop the index of the excitonic state. The energy- and momentumdependent cross section then can be written as

$$
\sigma\left(\omega_{i}, \omega_{f}, \boldsymbol{q}\right) \approx-\sum_{n}\left|\Lambda^{n}\left(\omega_{i}, \boldsymbol{q}\right)\right|^{2} \operatorname{Im} D^{n}\left(\omega_{i}-\omega_{f}, \boldsymbol{q}\right),
$$


where $\Lambda^{n}(\omega, \boldsymbol{q})$ is the complex off-diagonal part of the exciton Green's function, representing the scattering of an exciton by $n$ phonons of total momentum $\boldsymbol{q}=\boldsymbol{k}_{i}-\boldsymbol{k}_{f}$, and the presence of phonons in the final state is reflected by the many-body Green's function $D^{n}(\omega, \boldsymbol{q})$. In the limit of infinite phonon lifetime, $\operatorname{Im} D^{n}(\omega, \boldsymbol{q})=$ $-(1 / \pi) \delta\left(\omega-\sum_{i=1, n} \omega_{i}\right) \delta\left(\boldsymbol{q}-\sum_{i=1, n} \boldsymbol{q}_{i}\right)$. Here, we account for phonon-phonon interactions by attributing a small lifetime broadening to the phonon propagator.

Previous work suggests an approximate form of $\Lambda$ in the limits of weak [29] and intermediate [30] $e$-ph interactions. For the purpose of this work, however, we use a closedform solution for $\Lambda$ in the limit of local excitons coupled linearly to phonons. Although the cross section in Eq. (B1) is given in the energy domain, from here on we use the time-dependent form. Focusing on the characteristic behavior of the phonon satellites with multiple momentum contributions, we have

$$
\Lambda^{n}(t, \boldsymbol{q})=\Lambda^{0}(t) \prod_{\boldsymbol{q}_{i} \cdots \boldsymbol{q}_{n}}\left[i G\left(\boldsymbol{q}_{i}\right) \int_{0}^{t} D^{>}\left(\tau, \boldsymbol{q}_{i}\right) d \tau\right] / \sqrt{n !}
$$

The diagonal part of the exciton Green's function is denoted $\Lambda^{0}$ and is the same dressed propagator that would appear in the absorption process $\left(I_{\mathrm{XAS}} \sim \mathcal{F}\left\{\Lambda^{0}\right\}\right)$. The bracketed part of Eq. (B2) represents the phonon contributions to the scattering diagram, with the product over $n$ phonons conserving momentum, $\boldsymbol{q}=\boldsymbol{q}_{1}+\cdots+\boldsymbol{q}_{n} . G(\boldsymbol{q})$ is the momentum-dependent electron-phonon interaction strength, and the greater phonon propagator in the zerotemperature limit is simply $D^{>}(t, \boldsymbol{q})=-i\left\langle b_{\boldsymbol{q}}(t) b_{\boldsymbol{q}}^{\dagger}(0)\right\rangle=$ $i \theta(t) e^{-i \omega(\boldsymbol{q}) t}$. Thus, the nondiagonal part of the exciton Green's function differs from the diagonal part by the interaction term with a certain number of final state phonons. Despite the simple form, in the limit of small excitons, Eq. (B2) accounts for all types of diagrams including those with vertex corrections.

The exciton propagator dressed by the exciton-phonon interaction can be expressed as $\Lambda^{0}(t)=L(t) e^{C(t)}$, where $L$ is the bare exciton Green's function. We account for the exciton lifetime by including a damping factor $e^{-\gamma t / 2}$ in $L$. Note that this expression contains half of the inverse lifetime, $\gamma / 2$. We use a value $\gamma / 2=0.15 \mathrm{eV}$, slightly adjusted from the core-hole lifetime as determined by x-ray photoelectron spectroscopy measurements $(0.1 \mathrm{eV})$ [40] to account for the presence of the extra electron (see Supplemental Material [38]).

Finally, the cumulant function to second order in the exciton-phonon interaction can be written

$C(t)=[L(t)]^{-1} \int_{0}^{t} \int_{0}^{t} L\left(t-\tau_{1}\right) \Sigma_{\mathrm{FM}}\left(\tau_{1}-\tau_{2}\right) L\left(\tau_{2}\right) d \tau_{1} d \tau_{2}$, where the Fan-Migdal self-energy is $\Sigma_{\mathrm{FM}}=$ $-i \sum_{\boldsymbol{q}} G(\boldsymbol{q})^{2} L(t) D(t, \boldsymbol{q})$. Substituting the exciton and phonon Green's functions and taking the time integrals in Eq. (B3) analytically [49], we get $C(t)=\sum_{q} G(\boldsymbol{q})^{2} /$ $\left[\omega(\boldsymbol{q})^{2} N\right]\left[e^{-i \omega(\boldsymbol{q}) t}+i \omega(\boldsymbol{q}) t-1\right]$. It is important to notice that the summation runs over the whole Brillouin zone without restriction, since the total momentum transfer of the contributing diagrams is zero. From the form of Eqs. (B2) and (B3), it is clear that for any final state configuration there are contributions to the cross section from phonons of all momenta. Therefore, even the intensity of the one-phonon peak depends on $G(\boldsymbol{q})$ throughout the Brillouin zone, not just at the experimental $\boldsymbol{q}$ point.

To evaluate Eq. (B3), we use the TO phonon dispersion calculated with density functional perturbation theory. Brillouin zone integrations are performed using a reduced set of weighted momentum points which reflect the symmetries of the graphite structure [50]. Having calculated all the contributions to Eq. (B2) in the time domain, we can then perform a Fourier transform at the given incident photon energy and plug it into Eq. (B1) to obtain the RIXS cross section.

[1] O. Gunnarsson, M. Calandra, and J. E. Han, Colloquium: Saturation of Electrical Resistivity, Rev. Mod. Phys. 75, 1085 (2003).

[2] H. Fröhlich, On the Theory of Superconductivity: The OneDimensional Case, Proc. R. Soc. A 223, 296 (1954).

[3] J. Bardeen, L. N. Cooper, and J. R. Schrieffer, Theory of Superconductivity, Phys. Rev. 108, 1175 (1957).

[4] L. Pintschovius, Electron-Phonon Coupling Effects Explored by Inelastic Neutron Scattering, Phys. Status Solidi (b) 242, 30 (2005).

[5] S. Piscanec, M. Lazzeri, F. Mauri, A. C. Ferrari, and J. Robertson, Kohn Anomalies and Electron-Phonon Interactions in Graphite, Phys. Rev. Lett. 93, 185503 (2004).

[6] A.C. Ferrari, Raman Spectroscopy of Graphene and Graphite: Disorder, Electron-Phonon Coupling, Doping and Nonadiabatic Effects, Solid State Commun. 143, 47 (2007).

[7] S.-I. Tanaka, M. Matsunami, and S.-I. Kimura, An Investigation of Electron-Phonon Coupling via Phonon Dispersion Measurements in Graphite Using Angle-Resolved Photoelectron Spectroscopy, Sci. Rep. 3, 3031 (2013).

[8] J. Zhou, H. D. Shin, K. Chen, B. Song, R. A. Duncan, Q. $\mathrm{Xu}$, A. A. Maznev, K. A. Nelson, and G. Chen, Direct Observation of Large Electronphonon Interaction Effect on Phonon Heat Transport, Nat. Commun. 11, 6040 (2020).

[9] Z. Yao, C. L. Kane, and C. Dekker, High-Field Electrical Transport in Single-Wall Carbon Nanotubes, Phys. Rev. Lett. 84, 2941 (2000).

[10] D. Novko and M. Kralj, Phonon-Assisted Processes in the Ultraviolet-Transient Optical Response of Graphene, npj 2D Mater. Appl. 3, 48 (2019).

[11] F. Caruso, Nonequilibrium Lattice Dynamics in Monolayer $\mathrm{MoS}_{2}$, J. Phys. Chem. Lett. 12, 1734 (2021). 
[12] A. D. Wright, C. Verdi, R. L. Milot, G. E. Eperon, M. A. Pérez-Osorio, H. J. Snaith, F. Giustino, M. B. Johnston, and L. M. Herz, Electronphonon Coupling in Hybrid Lead Halide Perovskites, Nat. Commun. 7, 11755 (2016).

[13] T. Handa, T. Aharen, A. Wakamiya, and Y. Kanemitsu, Radiative Recombination and Electron-Phonon Coupling in Lead-Free $\mathrm{Ch}_{3} \mathrm{Nh}_{3} \mathrm{SnI}_{3}$ Perovskite Thin Films, Phys. Rev. Mater. 2, 075402 (2018).

[14] H. Yavaş, M. van Veenendaal, J. van den Brink, L. J. P. Ament, A. Alatas, B. M. Leu, M.-O. Apostu, N. Wizent, G. Behr, W. Sturhahn, H. Sinn, and E. E. Alp, Observation of Phonons with Resonant Inelastic X-Ray Scattering, J. Phys. Condens. Matter 22, 485601 (2010).

[15] W. S. Lee, S. Johnston, B. Moritz, J. Lee, M. Yi, K. J. Zhou, T. Schmitt, L. Patthey, V. Strocov, K. Kudo, Y. Koike, J. van den Brink, T. P. Devereaux, and Z. X. Shen, Role of Lattice Coupling in Establishing Electronic and Magnetic Properties in Quasi-One-Dimensional Cuprates, Phys. Rev. Lett. 110, 265502 (2013).

[16] J. J. Lee, B. Moritz, W. S. Lee, M. Yi, C. J. Jia, A. P. Sorini, K. Kudo, Y. Koike, K. J. Zhou, C. Monney, V. Strocov, L. Patthey, T. Schmitt, T.P. Devereaux, and Z.X. Shen, Charge-Orbital-Lattice Coupling Effects in the dd Excitation Profile of One-Dimensional Cuprates, Phys. Rev. B 89, 041104(R) (2014).

[17] S. Moser, S. Fatale, P. Krüger, H. Berger, P. Bugnon, A. Magrez, H. Niwa, J. Miyawaki, Y. Harada, and M. Grioni, Electron-Phonon Coupling in the Bulk of Anatase $\mathrm{TiO}_{2}$ Measured by Resonant Inelastic X-Ray Spectroscopy, Phys. Rev. Lett. 115, 096404 (2015).

[18] S. Fatale, S. Moser, J. Miyawaki, Y. Harada, and M. Grioni, Hybridization and Electron-Phonon Coupling in Ferroelectric $\mathrm{BaTiO}_{3}$ Probed by Resonant Inelastic X-Ray Scattering, Phys. Rev. B 94, 195131 (2016).

[19] S. Johnston, C. Monney, V. Bisogni, K. Zhou, R. Kraus, G. Behr, V. N. Strocov, J. Málek, S.-L. Drechsler, J. Geck, T. Schmitt, and J. van den Brink, Electron-Lattice Interactions Strongly Renormalize the Charge-Transfer Energy in the Spin-Chain Cuprate $\mathrm{Li}_{2} \mathrm{CuO}_{2}$, Nat. Commun. 7, 10563 (2016).

[20] D. Meyers et al., Decoupling Carrier Concentration and Electron-Phonon Coupling in Oxide Heterostructures Observed with Resonant Inelastic X-Ray Scattering, Phys. Rev. Lett. 121, 236802 (2018).

[21] M. Rossi, R. Arpaia, R. Fumagalli, M. M. Sala, D. Betto, K. Kummer, G. M. De Luca, J. van den Brink, M. Salluzzo, N. B. Brookes, L. Braicovich, and G. Ghiringhelli, Experimental Determination of Momentum-Resolved ElectronPhonon Coupling, Phys. Rev. Lett. 123, 027001 (2019).

[22] J. G. Vale, C. D. Dashwood, E. Paris, L. S. I. Veiga, M. Garcia-Fernandez, A. Nag, A. Walters, K. J. Zhou, I. M. Pietsch, A. Jesche, P. Gegenwart, R. Coldea, T. Schmitt, and D. F. McMorrow, High-Resolution Resonant Inelastic X-Ray Scattering Study of the Electron-Phonon Coupling in Honeycomb $\alpha-\mathrm{Li}_{2} \mathrm{IrO}_{3}$, Phys. Rev. B 100, 224303 (2019).

[23] L. Braicovich, M. Rossi, R. Fumagalli, Y. Peng, Y. Wang, R. Arpaia, D. Betto, G. M. De Luca, D. Di Castro, K. Kummer, M. M. Sala, M. Pagetti, G. Balestrino, N. B. Brookes, M. Salluzzo, S. Johnston, J. van den Brink, and G. Ghiringhelli, Determining the Electron-Phonon Coupling in Superconducting Cuprates by Resonant Inelastic X-Ray Scattering: Methods and Results on $\mathrm{Nd}_{1+x} \mathrm{Ba}_{2-x} \mathrm{Cu}_{3} \mathrm{O}_{7-\delta}$, Phys. Rev. Research 2, 023231 (2020).

[24] A. Geondzhian, A. Sambri, G. M. De Luca, R. Di Capua, E. Di Gennaro, D. Betto, M. Rossi, Y. Y. Peng, R. Fumagalli, N. B. Brookes, L. Braicovich, K. Gilmore, G. Ghiringhelli, and M. Salluzzo, Large Polarons as Key Quasiparticles in $\mathrm{SrTiO}_{3}$ and $\mathrm{SrTiO}_{3}$-Based Heterostructures, Phys. Rev. Lett. 125, 126401 (2020).

[25] J. Li, A. Nag, J. Pelliciari, H. Robarts, A. Walters, M. Garcia-Fernandez, H. Eisaki, D. Song, H. Ding, S. Johnston, R. Comin, and K.-J. Zhou, Multiorbital Charge-Density Wave Excitations and Concomitant Phonon Anomalies in $\mathrm{Bi}_{2} \mathrm{Sr}_{2} \mathrm{LaCuO}_{6+\delta}$, Proc. Natl. Acad. Sci. U.S.A. 117, 16219 (2020).

[26] Y. Y. Peng, A. A. Husain, M. Mitrano, S. X.-L. Sun, T. A. Johnson, A. V. Zakrzewski, G. J. MacDougall, A. Barbour, I. Jarrige, V. Bisogni, and P. Abbamonte, Enhanced Electron-Phonon Coupling for Charge-Density-Wave Formation in $\mathrm{La}_{1.8-x} \mathrm{Eu}_{0.2} \mathrm{Sr}_{x} \mathrm{CuO}_{4+\delta}$, Phys. Rev. Lett. 125, 097002 (2020).

[27] X. Feng, S. Sallis, Y.-C. Shao, R. Qiao, Y.-S. Liu, L. C. Kao, A. S. Tremsin, Z. Hussain, W. Yang, J. Guo, and Y.-D. Chuang, Disparate Exciton-Phonon Couplings for ZoneCenter and Boundary Phonons in Solid-State Graphite, Phys. Rev. Lett. 125, 116401 (2020).

[28] L. J. P. Ament, M. van Veenendaal, and J. van den Brink, Determining the Electron-Phonon Coupling Strength from Resonant Inelastic X-Ray Scattering at Transition Metal L-Edges, Europhys. Lett. 95, 27008 (2011).

[29] T. P. Devereaux, A. M. Shvaika, K. Wu, K. Wohlfeld, C. J. Jia, Y. Wang, B. Moritz, L. Chaix, W.-S. Lee, Z.-X. Shen, G. Ghiringhelli, and L. Braicovich, Directly Characterizing the Relative Strength and Momentum Dependence of ElectronPhonon Coupling Using Resonant Inelastic X-Ray Scattering, Phys. Rev. X 6, 041019 (2016).

[30] A. Geondzhian and K. Gilmore, Demonstration of Resonant Inelastic X-Ray Scattering as a Probe of Exciton-Phonon Coupling, Phys. Rev. B 98, 214305 (2018).

[31] A. Geondzhian and K. Gilmore, Generalization of the Franck-Condon Model for Phonon Excitations by Resonant Inelastic X-Ray Scattering, Phys. Rev. B 101, 214307 (2020).

[32] K. Bieniasz, S. Johnston, and M. Berciu, Beyond the SingleSite Approximation Modeling of Electron-Phonon Coupling Effects on Resonant Inelastic X-Ray Scattering Spectra, SciPost Phys. 11, 62 (2021).

[33] M. Lazzeri, S. Piscanec, F. Mauri, A. C. Ferrari, and J. Robertson, Phonon Linewidths and Electron-Phonon Coupling in Graphite and Nanotubes, Phys. Rev. B 73, 155426 (2006).

[34] C.-H. Park, F. Giustino, M. L. Cohen, and S. G. Louie, Electron-Phonon Interactions in Graphene, Bilayer Graphene, and Graphite, Nano Lett. 8, 4229 (2008).

[35] M. X. Na, A. K. Mills, F. Boschini, M. Michiardi, B. Nosarzewski, R. P. Day, E. Razzoli, A. Sheyerman, M. Schneider, G. Levy, S. Zhdanovich, T. P. Devereaux, A. F. Kemper, D. J. Jones, and A. Damascelli, Direct Determination of Mode-Projected Electron-Phonon Coupling in the Time Domain, Science 366, 1231 (2019). 
[36] M. Mohr, J. Maultzsch, E. Dobardžić, S. Reich, I. Milošević, M. Damnjanović, A. Bosak, M. Krisch, and C. Thomsen, Phonon Dispersion of Graphite by Inelastic X-Ray Scattering, Phys. Rev. B 76, 035439 (2007).

[37] A. G. Marinopoulos, L. Reining, A. Rubio, and V. Olevano, Ab Initio Study of the Optical Absorption and Wave-VectorDependent Dielectric Response of Graphite, Phys. Rev. B 69, 245419 (2004).

[38] See Supplemental Material at http://link.aps.org/ supplemental/10.1103/PhysRevX.11.041052 for details of self-absorption corrections, phonon mode assignments, and further data and fitting.

[39] P. A. Brühwiler, A. J. Maxwell, C. Puglia, A. Nilsson, S. Andersson, and N. Mårtensson, $\pi^{*}$ and $\sigma^{*}$ Excitons in C 1s Absorption of Graphite, Phys. Rev. Lett. 74, 614 (1995).

[40] F. Sette, G. K. Wertheim, Y. Ma, G. Meigs, S. Modesti, and C. T. Chen, Lifetime and Screening of the C 1 s Photoemission in Graphite, Phys. Rev. B 41, 9766 (1990).

[41] W. Olovsson, T. Mizoguchi, M. Magnuson, S. Kontur, O. Hellman, I. Tanaka, and C. Draxl, Vibrational Effects in X-Ray Absorption Spectra of Two-Dimensional Layered Materials, J. Phys. Chem. C 123, 9688 (2019).

[42] J. Jiang, R. Saito, K. Sato, J. S. Park, G. G. Samsonidze, A. Jorio, G. Dresselhaus, and M. S. Dresselhaus, ExcitonPhoton, Exciton-Phonon Matrix Elements, and Resonant
Raman Intensity of Single-Wall Carbon Nanotubes, Phys. Rev. B 75, 035405 (2007).

[43] G. Antonius and S. G. Louie, Theory of the Exciton-Phonon Coupling, arXiv:1705.04245.

[44] H.-Y. Chen, D. Sangalli, and M. Bernardi, Exciton-Phonon Interaction and Relaxation Times from First Principles, Phys. Rev. Lett. 125, 107401 (2020).

[45] M. P. M. Dean et al., Ultrafast Energy- and MomentumResolved Dynamics of Magnetic Correlations in the Photo-doped Mott Insulator $\mathrm{Sr}_{2} \mathrm{IrO}_{4}$, Nat. Mater. 15, 601 (2016).

[46] Y. Cao, D. G. Mazzone, D. Meyers, J. P. Hill, X. Liu, S. Wall, and M. P. M. Dean, Ultrafast Dynamics of Spin and Orbital Correlations in Quantum Materials: An Energyand Momentum-Resolved Perspective, Phil. Trans. R. Soc. A 377, 20170480 (2019).

[47] M. Mitrano and Y. Wang, Probing Light-Driven Quantum Materials with Ultrafast Resonant Inelastic X-Ray Scattering, Commun. Phys. 3, 184 (2020).

[48] D. G. Mazzone et al., Laser-Induced Transient Magnons in $\mathrm{Sr}_{3} \mathrm{Ir}_{2} \mathrm{O}_{7}$ throughout the Brillouin Zone, Proc. Natl. Acad. Sci. U.S.A. 118, e2103696118 (2021).

[49] D. C. Langreth, Singularities in the X-Ray Spectra of Metals, Phys. Rev. B 1, 471 (1970).

[50] H. J. Monkhorst and J. D. Pack, Special Points for BrillouinZone Integrations, Phys. Rev. B 13, 5188 (1976). 